\title{
A Method to Perform a Fast Fourier Transform With Primitive Image Transformations
}

\author{
Phil Sheridan, Member, IEEE
}

\begin{abstract}
The Fourier transform is one of the most important transformations in image processing. A major component of this influence comes from the ability to implement it efficiently on a digital computer. This paper describes a new methodology to perform a fast Fourier transform (FFT). This methodology emerges from considerations of the natural physical constraints imposed by image capture devices (camera/eye). The novel aspects of the specific FFT method described include: 1) a bit-wise reversal re-grouping operation of the conventional FFT is replaced by the use of lossless image rotation and scaling and 2) the usual arithmetic operations of complex multiplication are replaced with integer addition. The significance of the FFT presented in this paper is introduced by extending a discrete and finite image algebra, named Spiral Honeycomb Image Algebra (SHIA), to a continuous version, named SHIAC.
\end{abstract}

Index Terms-Euclidean ring, hexagonal lattice, image transforms.

\section{INTRODUCTION}

$\mathbf{T}$ HE Fourier transform is one of the most important transformations in image processing in particular and signal processing in general. Its origins date back to 1807 when Jean Baptiste Joseph Fourier defined the notion of representing a function as a trigonometric series. Until the invention of the digital computer, the Fourier series was employed as a purely analytic tool. However, with the development of the fast Fourier transform (FFT), the notion has become a useful computational tool. One of the most attractive computational properties of the FFT is its ability to process signals at higher resolution with a minimal increase in cost to complexity. Today, most of us use FFTs every day, without even knowing it, as this technology is employed in digital cameras, disc drives, and even our cell phones [1]. Since its original inception in the early 1960s, the FFT has undergone a multitude of mutations many of which have resulted in patents. The motivation behind the development of these FFT variants is the commercial advantage gained by achieving rapid creation of a Fourier signal and a rapid reconstruction of the original signal from it.

Primitive image transformations, translation, rotation, and scaling, are fundamental to many image processing tasks

Manuscript received January 15, 2006; revised October 25, ,2006. The associate editor coordinating the review of this manuscript and approving it for publication was Dr. Giovanni Ramponi.

The author is with the School of computing and information technology, Griffith University, Meadowbrook, QLD 4131 Brisbane, Australia (e-mail: p.sheridan@griffith.edu.au).

Color versions of one or more of the figures in this paper are available online at http://ieeexplore.ieee.org.

Digital Object Identifier 10.1109/TIP.2007.891790 because they mimic object motion in the visual world. Image representations, which are invariant to such motion, have added utility to both biological and computer vision systems. Some of the most important image processing properties of the Fourier transform result from their pseudo invariance to the primitive object motion of the objects they represent. Hence, it is not surprising that the Fourier transform has a strong relation to mathematical structures composed of pseudo invariant image transformations. The Spiral Honeycomb Image Algebra (SHIA) is one such mathematical structure that represents images on a hexagonal lattice. In [2], it was shown that this class of algebras is important to the field of image processing and computer vision for two reasons. First, it facilitates lossless image transformations for rotation, scaling and translation. Second, it possesses computational properties pertinent to discrete representations of images, digital image technology, and biological vision.

The main thrust of this paper investigates extensions to SHIA and serves the purpose of proposing a new methodology for performing image transformations; one which is consistent with the constraints imposed by image capture devices. Two outcomes will be described: 1) a new theoretical framework that facilitates pattern analysis and computer vision; 2) a new method to perform a FFT. The first outcome emerges from a distinction between the environment in which objects move about (referred to in this paper as the visual world) and the portion of the environment visible to an observer at any given point in time due to the physical constraints of the camera/eye of the observer (referred to in this paper as the visual field). It will be shown that SHIA captures important aspects of the visual field that are unobtainable by other mathematical structures currently employed to support image analysis. The extensions to SHIA will also reveal SHIA's relationship to the most powerful mathematical structure (complex plane) commonly employed to represent the visual world. The second outcome describes a new FFT algorithm of type Cooley-Tukey which is peculiar to SHIA. It will be shown that this FFT named SHIA-FFT is in general comparable in efficiency to other FFTs but possesses additional efficiency in certain circumstances.

The FFT presented in this paper is similar to two other FFTs defined on a hexagonal lattice. Zapata and Ritter [3] proposed an FFT defined on the Generalized Balanced Tree. Middleton [4] described a FFT, named HIP-FFT, on an image represented on a hexagonal lattice. Middleton's paper makes two important contributions to the theory of FFTs: 1) it provides empirical evidence for the quality of the Fourier transform when performed on a hexagonal lattice; 2) it provides the mathematical motivation underpinning the FFT on the hexagonal lattice. 
SHIA-FFT distinguishes itself from HIP-FFT in two regards. First, the HIP-FFT employs a third coordinate system to relate the addresses on the hexagonal lattice to the re-grouping process. The SHIA-FFT achieves this regrouping with the use of a lossless primitive image transformation from SHIA. Second, and as a consequence of the extension of SHIA to a continuous domain, the arithmetic operations of complex multiplication and addition are performed in SHIA in integer arithmetic without resorting to the usual operations within the field of complex numbers. It will be shown in this paper that performing arithmetic operations in SHIA, instead of the complex field, can result in considerable computational savings at the cost of incurring a user-defined degree of approximation.

Currently, SHIA is defined on a finite set of points and possesses the powerful mathematical structure of a Euclidean ring. However, it falls short of the important mathematical structure of a field by its lack of multiplicative commutativity. This paper extends SHIA from a discrete and finite domain to a continuous domain. A consequence of this extension is that the continuous version of SHIA named SHIAC achieves the structure of a field. More specifically, it is shown that SHIAC is isomorphic to the field of complex numbers. It is a consequence of this isomorphism that permits the otherwise complex arithmetic operations to be performed in the computational environment of SHIA.

Section II of this paper provides a brief review of the fundamental properties of the mathematical structure from which all the results of this paper emerge. Section III provides a description of a new algorithm to perform the FFT named SHIA-FFT and which is based on the SHIA properties presented in the previous section. Section IV describes two extensions to SHIA. Section IV-A extends the discrete and finite nature of SHIA to a continuous domain where it is shown that this extension possesses all of the properties of the complex plane. Section IV-B shows how to perform all of the computations required for an FFT with SHIA's arithmetic operations. A case study presented in Section IV-C describes a method to measure the computational advantages of performing the FFT in SHIA. Section IV-D describes the minimal set of alterations to SHIA so that all of its computational properties can be applied to signals sampled by a rectangular lattice. Section $\mathrm{V}$ outlines a method that employs SHIA to perform an FFT in purely integer arithmetic and the circumstances where this would be an advantage to do so. Section VI presents a formal statement of three algorithms discussed in this paper. Section VII presents the conclusions of this paper.

\section{SpIRAl Honeycomb IMAge Algebra (SHIA)}

The distribution of photoreceptors on the primate's retina and the distribution of cells in the human cortex [5], [6] are highly suggestive of primitive image transformations associated with the vision process. A natural data structure that emerges from geometrical considerations of this distribution is the SHIA. This class of algebras was initially described in [2] and further extended in [7] and [8]. However, as these results are critical to the core results of this paper, we present a brief review of the fundamental issues associated with the SHIA in this section.

The underlying geometry of the SHIA is a hexagonal lattice, with each hexagon having a designated positive integer address expressed in base seven. The numbered hexagons form clusters of super hexagons of size $7^{n}$. These self-similar super-hexagons tile the plane in a recursively modular manner. As an example, a super-hexagon of size $7^{2}$ and its concomitant addressing scheme is displayed in Fig. 1(a).

This addressing scheme has associated with it a collection of transformations, which form a powerful mathematical structure known as a Euclidean ring. This mathematical structure possesses almost all the algebraic properties normally attributed to the real and complex number systems. The SHIA algebraic structure emerges from two arithmetic operations defined on its addressing scheme, spiral addition, and spiral multiplication. Each of these arithmetic operations defines a transformation on its address space. When a super-hexagon also has associated with it a discrete sampling of a 2-D signal, the arithmetic operations take on a geometrical interpretation of the sampled signal. The spatial information embodied in the signal is captured algebraically by the operations. Spiral addition is associated with translation of the signal, and spiral multiplication is associated with the dual transformations of rotation and scaling. A critical property of these transformations is that no information of the originally sampled signal is ever lost under the action of the transformations. An explanation for this property and a full discussion of the SHIA is provided in [2].

The transformation from SHIA pertinent to the FFT as discussed in this paper is denoted by M10. The symbol "M" in this notation refers to the application of spiral multiplication to the address in SHIA that follows it - the number 10 in this case. Although the full definition of spiral multiplication, denoted by the symbol $\otimes$, is given in the Appendix, we can conveniently define here the spiral multiplication of an address in SHIA by address 10 as the anti clockwise permutation of the digits in its representation. That is, let $a=a_{n} a_{n-1} \ldots a_{1} a_{0}$ represent an address in a $7^{n+1}$ hexagonal SHIA, where $a_{i}[0, \ldots, 6]$ for $\mathrm{i}=0$ to $\mathrm{n}$. Then

$$
10 \otimes a=a_{n-1} a_{n-2}, \ldots, a_{0} a_{n} .
$$

For example, in a 49-hexagonal SHIA where each address is represented by two digits, $10 \otimes 01=10$ and $10 \otimes 10=01$. The relation between the address space of Fig. 1(a) and (b) is exactly that of M10.

In a similar manner, the inverse operation to M10 is obtained by performing a rotation of the digits in the opposite direction to that which was performed to obtain M10.

Fig. 2(a)-(d) displays the effect of M10 when applied to an image represented on an SHIA of size $7^{4}=2401$. Each of the four images can be derived from successive applications of either M10 or its inverse to any of the other images displayed in the figure. In other words, M10 and its inverse perfectly recover pixel information of the originally sampled image from any of its transformations.

Another property of M10, which is observable in the images displayed in Fig. 2, is that each transformation assembles the image at a unique resolution. Fig. 2(a) shows multiple views of the image at the lowest possible nontrivial resolution. That is, the image is composed of 343 subimages, each of which is composed of seven equally spaced pixels from the input image. 


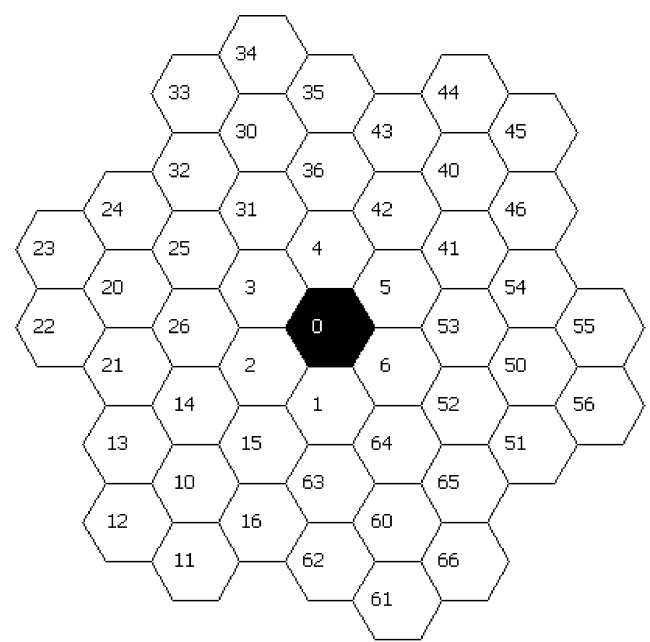

(a)

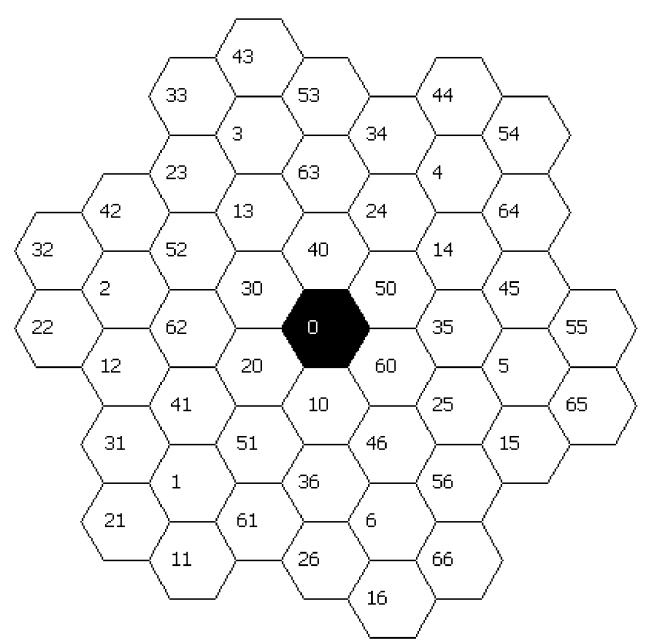

(b)

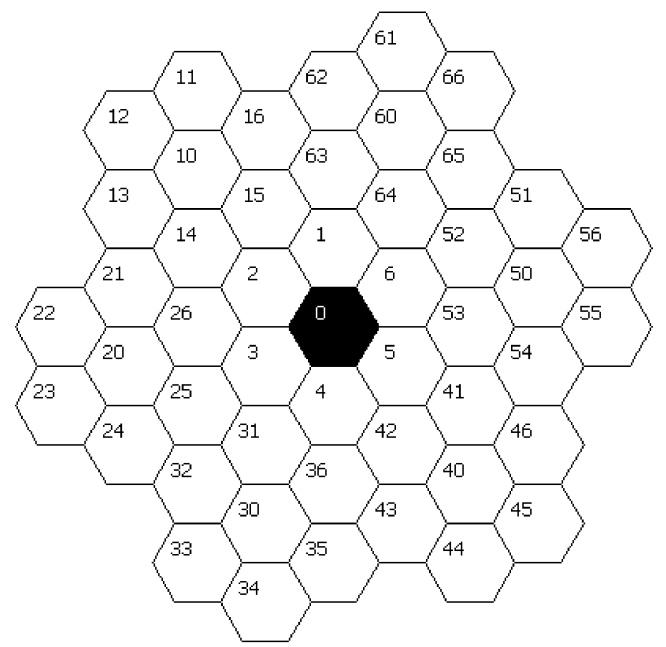

(c)

Fig. 1. (a) Address space of a $7^{2}=49$ Hexagon SHIA; (b) results from applying M10 of SHIA to (a); (c) frequency domain associated with the spatial domain as displayed in (a).

If M10 is applied to this image, the resultant image is that displayed in Fig. 2(b), one that is composed of 49 subimages of size $7^{2}$. Successive applications of M10 reduce the number of subimages by a factor of seven and increase the resolution of

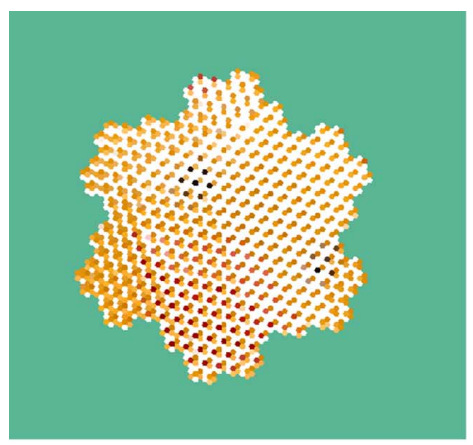

(a)

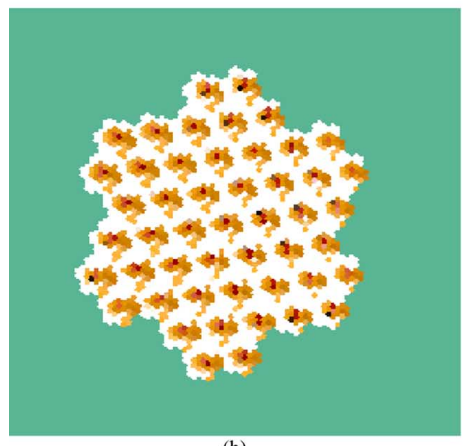

(b)

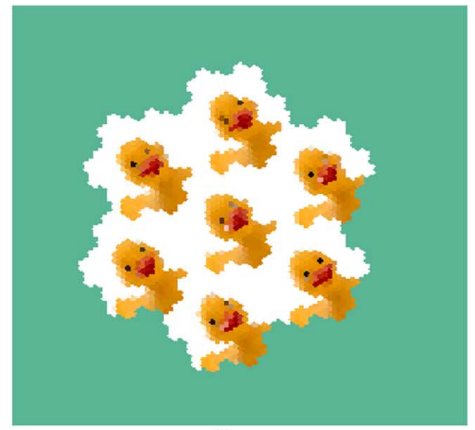

(c)

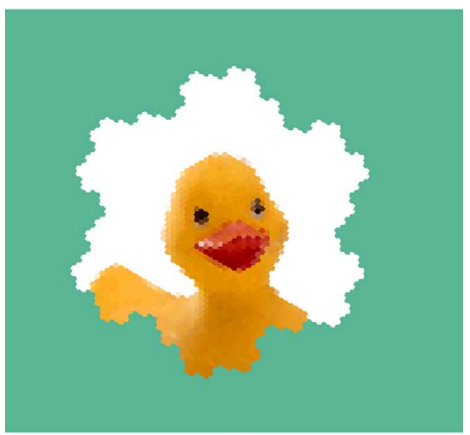

Fig. 2. Four transformations of an image represented on an SHIA of size $7^{4}=$ 2401 pixels. (a) Result of M10 applied to (d); (b) result of applying M10 to (a); (c) result of applying M10 to (b); (d) input image and also results from the application of M10 to (c).

each subimage by the same factor. Once the image of Fig. 2(d) is achieved, one subimage of size $7^{4}=2401$, a further application of M10 results in the image displayed in Fig. 2(a). In this particular case, the modular aspect of the M10 has produced the identical effect of applying the inverse of M10 to Fig. 2(b).

It is exactly this lossless recovery property and the particular partitioning property of M10 that facilitates the efficient regrouping of the computations of the FFT to be described in 
this paper. This point will be returned to and explained in the following section of this paper.

A final important point to address before we consider the FFT on SHIA is the relation between the lattices of the spatial domain and the frequency domain. As was reported in [3] and [9], the relation can be described as reciprocal. That is the spatial and frequency axis is orthogonal to one another. Fig. 1(c) illustrates this relation where it can be observed that the frequency domain is a rotation of the spatial domain plus a reflection about its vertical axis. The mathematical derivation of this relation, provided in [3] and [9], also indicates a scaling between the two domains. The important point concerning this relation is that it is crucial for the appropriate interpretation of the resulting Fourier transform.

\section{FFT IN SHIA}

The FFT distinguishes itself from the Fourier transform, commonly known as discrete Fourier transform (DFT), by the order in which the computations of the complex arithmetic operations, addition and multiplication, are performed. The DFT for a 1-D signal is defined as

$$
F(u)=\frac{1}{N} \sum_{x=0}^{x=N-1} f(x) e^{-j 2 \pi x u / N}
$$

for $u=0, \ldots, N-1$, where $f(x)$ is a real value function, $N$ represents the number of elements in the signal and $\mathrm{j}^{2}=-1$.

A casual analysis of (2) reveals that the DFT algorithm is of order $O\left(N^{2}\right)$ in the number of complex arithmetic operations performed. In contrast, a Cooley-Tukey type FFT regroups the complex operations to achieve a $O(N \log (N))$ algorithm. The SHIA-FFT algorithm is of type Cooley-Tukey and is described in Section III-A. Section III-B provides an analysis of the algorithm. A proof of the algorithm's correctness is presented in the appendix.

\section{A. Informal Statement of Algorithm}

Initially, the sampled input signal is represented on an SHIA of an appropriate size. For the purposes of the discussion in this section, we will assume that the size $N=7^{4}$ is the image depicting the duck of Fig. 2(d). Denote this collection of values as $f(x)$. Note that although $f(x)$ is a function of a single variable, it represents a 2-D signal. A three-step process: 1) localization, 2) discrete Fourier transform, and 3) globalization is applied $4=\log _{7}\left(7^{4}\right)$ times. We refer to each of the repetitions of the three-step process as a level of processing.

1) Level 1: At the first level of processing, the algorithm performs a DFT at each of $343=7^{3}$ subimages each of which is of size 7. This is achieved by applying each of the three steps mentioned above. First, the localization process is achieved by applying M10 to $f(x)$. The result is to transform the original spatial domain into that which is observable in Fig. 2(a). In other words, the effect is a regrouping of the spatial domain at the lowest resolution. Second, the DFT is performed on each of the 343 subimages of size 7 . Third, the globalization process is applied which in this case is the identity map. The lowest level of the FFT is complete as seen in Fig. 3(a).
2) Level 2: At the second level of processing, the effect of the algorithm is to produce $49=7^{2}$ Fourier transforms of the 49 subimages as displayed in Fig. 2(b). At this level, the localization process has two components: 1) M10 is applied to the resulting frequency domain from the previous level; 2) ss the subimages at this level are of size 49; the 49 addresses associated with the 49 roots of unity are mapped into an address space. Then, M10 is applied to this address space so that the appropriate roots of unity line up in packets of seven with the transformed signal. The effect of M10's application to the new spatial domain is a re-grouping, in clusters of seven, at the next higher level of resolution. Likewise, the effect of M10's application to the roots of unity is a re-grouping, in clusters of seven, of the unit circle into seven clusters, each of which contains seven addresses of equal and maximal spacing on the unit circle. At this stage, the localization process is complete and the DFT on each of the seven clusters is performed. In order to complete the three-step process at this level, the globalization process is then performed. This amounts to applying the inverse of M10 on each super-hexagon of size 49. The effect of this transform is a re-grouping of the frequency domain into consecutive super-hexagons at the second lowest resolution; subimages of size 49. This result is observable in Fig. 3(b).

At each of the two remaining subsequent levels, this same three-step process of localization, discrete Fourier transform and globalization is performed. The results at each level achieve the Fourier transform at the next higher level of resolution, the results of which are presented in Fig. 3(c) and (d).

\section{B. Analysis of Algorithm}

The standard analysis of a Fourier transform is to count the number of complex arithmetic operations performed. All other computations are considered overhead and ignored. In the worst case, M10 is linear in integer addition. In practice and as a result of the need for repeated applications of this transformation, M10 can be preprocessed and held in memory. Consequently, the costs associated with M10 are also ignored.

At each local execution of the DFT, the number of complex multiplication operations required is $7^{2}$. There are also an equal number of complex addition operations. We denote the number of sampled points to be transformed as $\mathrm{N}=7^{n}$, where $n$ is a positive integer. It follows that the number of times that the local DFT is performed at each level is $7^{n-1}$. The number of complex operations at each level is $2\left(7^{2}\right)\left(7^{n-1}\right)$. As the number of levels is $\log _{7}\left(7^{n}\right)=n$, it follows that the total number of operations to be performed is $2\left(7^{2}\right)\left(7^{n-1}\right) n$. Therefore, SHIA-FFT is of order $O(N \log (N))$ in the number of complex operations of multiplication to be performed. In the following sections, we extend SHIA and show how the most expensive operation of complex multiplication can be replaced by the relatively inexpensive operation of integer addition and thereby achieve an FFT, which is $O(N \log (N))$ in integer addition.

\section{EXTENSION TO SHIA}

Although SHIA was originally conceived as a collection of lossless primitive image transformations of translation, rotation and scaling, it also has a strong relation to complex numbers. In particular, the address space of SHIA constitutes a subset 


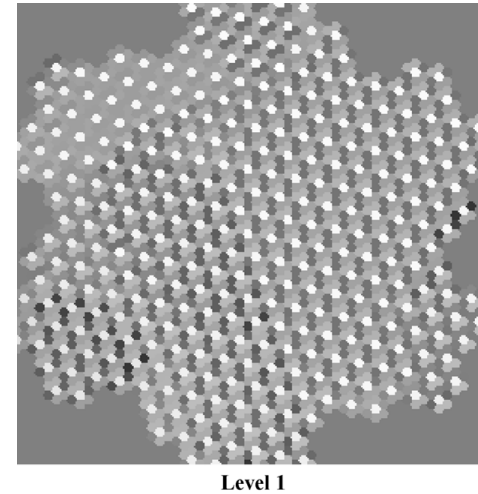

(a)

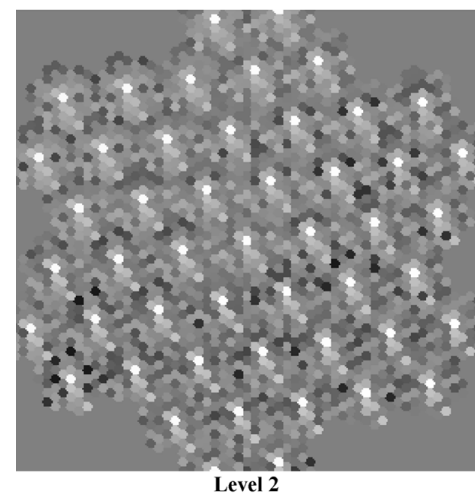

(b)

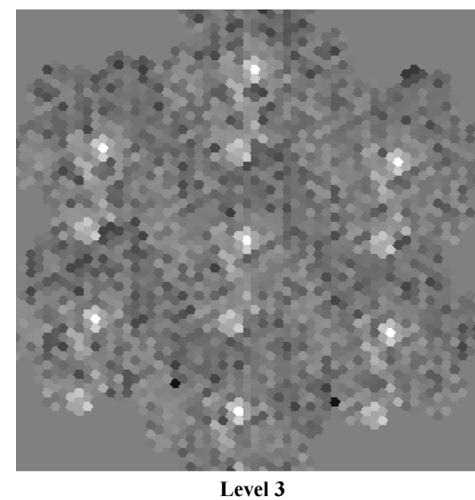

(c)
Level

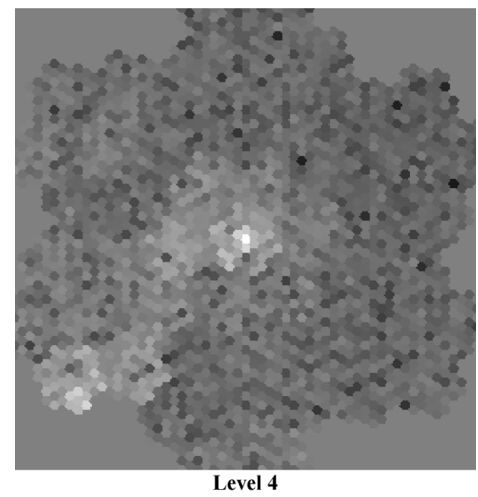

Fig. 3. Four stages of the Fourier transform of the image displayed in Fig. 2(d). (a) Result on completion of level 1; (b) result on completion of level 2; (c) result on completion of level 3; (d) completed transform level 4.

of the complex plane and spiral addition and spiral multiplication mimic their counterpart operations of complex addition and complex multiplication. This relation can be strengthened to an isomorphism [10]. This paper will develop the isomorphism by making two alterations to SHIA that will shortly be described. However, before wading into the mathematical details of the task, it is worth considering the theoretical and computational motivation for establishing the isomorphism. As a consequence of this isomorphism: 1) the relationship between the visual world and the visual field will be described in mathematical terms, and 2) we will show how the FFT can be performed with purely integer arithmetic. That is, the usual floating point operations associated with the multiplication and addition of complex numbers can be dispensed with by performing spiral multiplication and spiral addition on the isomorphic image of these complex numbers. Moreover and as a further consequence of the isomorphism, we will show how the computation of a multiplication operation on complex numbers can be performed at the computational cost of integer addition. This means that we will be able to replace the most computationally expensive operation, multiplication of the FFT, with the relatively inexpensive operation of integer summation. With this motivation now established, we consider the mathematical details of the isomorphism.

\section{A. Continuous Spiral Honeycomb Image Algebra SHIAC}

Up to this point in the discussion, SHIA consists of a finite set of addresses, the cardinality of which is $7^{n}$, where $n<\infty$. As stated earlier in this paper, SHIA has the mathematical structure of a Euclidean ring. The mathematical proofs that the arithmetic operations of spiral addition and spiral multiplication defined on SHIA possess all the required algebraic properties (e.g., associative, distributive, etc.) are provided in [11]. However, SHIA, a Euclidean ring, falls short of the mathematical structure of a field by virtue of the fact that its operation of modular spiral multiplication is not fully commutative. In particular, the multiplication of addresses that are a power of 7 with addresses that are not a power of 7 does not commute. In this section, we will extend the address space of SHIA to a possibly infinite set and remove the modular component from the multiplication operation. By doing so, this extension becomes a field. Then we will show that this extension to SHIA is isomorphic to the field of complex numbers.

1) Extending the Address Space of SHIA: An address in SHIA is represented as $a=\sum_{k=0}^{n} a_{k} 7^{k}$ where $a_{k} \in[0, \ldots, 6]$. This purely integer representation of an address can be generalized to incorporate floating point addresses by starting the index variable, $k$, at a negative integer

$$
a=\sum_{k=-m}^{n} a_{k} 7^{k}
$$

where $a_{k} \in[0,1,2,3,4,5,6]$ and $m, n \geq 0$.

With (3), we can now define the address space of the SHIAC.

Definition: Let SHIAC $(m, n)$ be the set of all addresses defined in (3) for integer values of $m$ and $n$. In this space, the values of $m$ and $n$ may be either infinite or finite.

As a simple example of this addressing scheme, the address space of SHIAC $(1,1)$ is displayed in Fig. 4. 


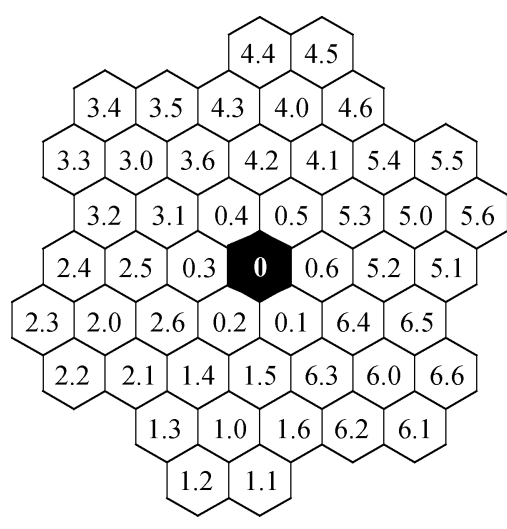

Fig. 4. The 49 addresses of $\operatorname{SHIAC}(1,1)$.

The second change to SHIA that is required is to the definition of multiplication. The need arises due to the fact that when either of the values of $m$ or $n$ in SHIAC ( $m, n)$ is not finite, the notion of modular spiral multiplication and modular spiral addition is inapplicable. As a consequence, we re-define the definition of the multiplication in SHIAC to be spiral multiplication, as presented in the Appendix. The consequence of removing the modular component of the multiplication operation is that spiral multiplication without modularity is commutative. The immediate consequence of gaining commutativity in the multiplication operation is that all the axioms that define the mathematical structure known as a field now hold for SHIAC.

It is asserted that SHIAC $(m, n)$ is a field when $m$ and $n$ are not finite. The mathematical proofs of the spiral addition and spiral multiplication defines a field follows from the arguments presented in [11].

As a consequence of the assertion, SHIAC has all the algebraic properties of the complex plane. In the following section, it will be shown that up to the way in which the operations of addition and multiplication are performed SHIAC and the complex plane are the same.

2) Mapping Shiac to the Complex Plane: Having established the fact that the representation for the addresses of SHIAC together with the operations of spiral addition and spiral multiplication form a field, we next define a mapping from SHIAC into the complex plane $(C)$ as originally reported in [10] and show that this mapping is an isomorphism. To this end, we use the fact that the set of addresses $\left\{7^{i}\right\}$ for $i \in[-m, n]$ form a basis for SHIAC $(m, n)$. We associate with each address of the basis, a pair of real numbers that represent its Euclidean distance from the address 0 and the angle it subtends with the vertical axis through address 0 . This means that the mapping $\tau\left(7^{i}\right) \Rightarrow\left(r_{i}, \theta_{i}\right)$ where $\left(r_{i}, \theta_{i}\right)$ assigns to each address of the basis, a point in the complex plane represented in polar coordinates. In order to determine the explicit value of $\left(r_{i}, \theta_{i}\right)$, consider the relation between the addresses 10 and 1 in SHIAC as displayed in Fig. 5.

In the figure, $x=2, y=1, \phi=2 \pi / 3$. From the Law of Cosines

$$
\begin{aligned}
r^{2} & =x^{2}+y^{2}-2 x y \cos (120)=4+1+2=7 \\
r & =\sqrt{7} \\
\theta & =\cos ^{-1}\left(\frac{7-4-1}{-4}\right)=\cos ^{-1}(-.5) .
\end{aligned}
$$

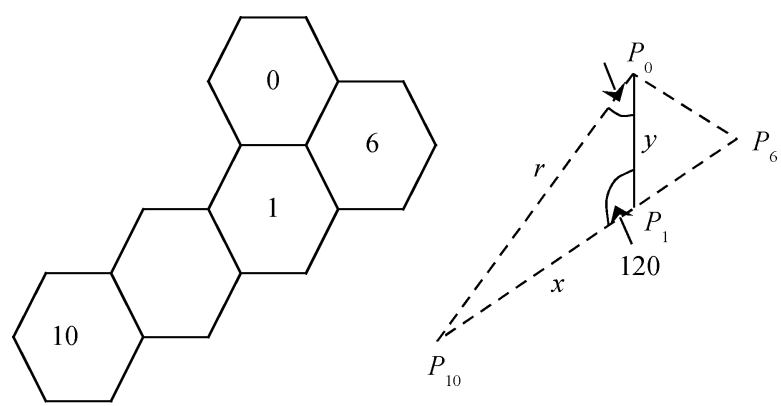

a)

b)

Fig. 5. (a) Geometrical relation between the hexagons containing the addresses $0,1,6$ and 10 in SHIAC; (b) $P_{i}$ denotes the point in the complex plane $C$ that corresponds to the center of hexagon of address $i$. The four points define three triangles from which the mapping from the hexagons of SHIAC to the points of $C$ is derived.

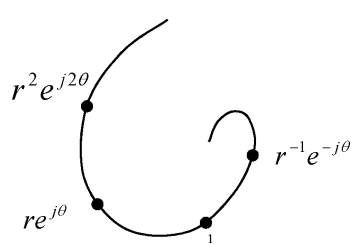

Fig. 6. Graphical representation of the spiral $f(i)=r^{i} e^{j i \theta}$. The integer values of $i$ relate the hexagonal addresses of SHIAC to the polar coordinates of the point in the center of hexagon of address $i$.

More generally, for any integer value $i$, the distance of address $7^{i}$ to the origin is $(\sqrt{7})^{i}$ and the angle associated with address $7^{i}$ is $i \theta$. This means that the ratio of distances of address $7^{i}$ and $7^{i-1}$ is the constant $r$ and the angle subtended by these addresses with the origin is the constant $\theta$ for all integer values of $i$. Fig. 6 displays a graph of the equation $f(i)=r^{i} e^{j i \theta}$ The addresses in SHIAC associated with the points along the curve for integer values of $i$ constitute the basis of SHIAC and it is this spiral from which the "S" in SHIA is derived.

From these relations, we define a mapping from SHIAC to the complex plane as follows:

$$
\begin{aligned}
\psi(\text { SHIAC }) & \rightarrow C \\
\psi\left(\sum_{k=-m}^{n} a_{k} 7^{k}\right) & \Rightarrow \sum_{k=-m}^{n} \delta r^{k} e^{j \pi a_{k}(2 \theta+(1 / 3))}
\end{aligned}
$$

where $\delta=0$ if $a_{k}$ is 0 and 1 otherwise.

As a consequence of the mapping $\psi$, it can also be observed that as $m, n \rightarrow \infty$, hexagons of SHIAC $(m, n)$ converges to points in the complex plane. In practice, this mapping can also be used to determine the amount of error embodied in a particular approximation of an address in SHIAC under its mapping for particular values of $m$ and $n$. In particular, for any given tolerance of error, $\varepsilon$, a minimal value for $m$ that guarantees that a point in SHIAC $(m, n)$ approximates a desired point in the complex plane within that tolerance is given by the following inequality:

$$
m>\frac{\log (\varepsilon)}{\log (\sqrt{7})} .
$$




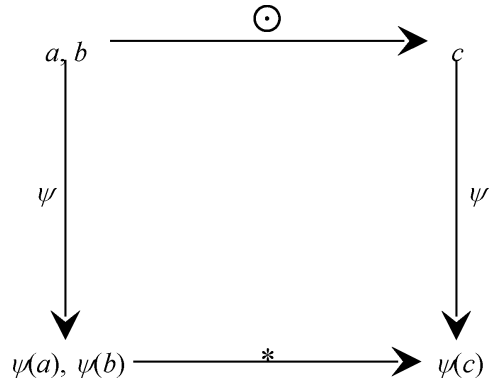

Fig. 7. Isomorphic relation of the mapping $\psi$, when $\odot$ represents the operation in SHIAC and $*$ represents the operation in the complex plane.

Having established that under the mapping $\psi$ any number in the complex plane can be represented in SHIAC, and vice versa, it is asserted that the mapping $\psi$ is one-to-one and on-to. It can also be shown, albeit algebraically tediously, that the mapping is associative. That is, the relation displayed in Fig. 7 holds for all addresses in SHIAC. To demonstrate the associativity of the mapping, consider the result of multiplying the address 10 by itself before and after the mapping

$$
\begin{gathered}
\psi(10 \otimes 10)=\psi(100) \Rightarrow\left(r^{2}, 2 \theta\right) \\
\psi(10) \psi(10) \Rightarrow(r, \theta) \bullet(r, \theta)=\left(r^{2}, 2 \theta\right) .
\end{gathered}
$$

As a consequence of the above-mentioned algebraic properties of SHIAC and $\psi$, it is asserted that the fundamental theorem of SHIAC follows.

Theorem 1: SHIAC is isomorphic to the complex field as defined by the mapping $\psi$ of (4).

Apart from the theoretical importance of the isomorphism, there are practical implications, as well. As an example of one such application of the isomorphism, a simple algorithm for determining the multiplicative inverse of an address of SHIAC can be derived.

Denote the multiplicative inverse of address $\alpha$, by $\alpha^{-1}$. In the context of a continuous address space, it also makes sense to employ the fraction symbol to denote the inverse, i.e., $1 / a$, because the way in which we calculate the multiplicative inverse in SHIAC is analogous to the process of determining the multiplicative inverse in the field of real numbers. That is, the multiplicative inverse of a real number can be calculated by performing the usual long division algorithm on 1 by the given real number. Recall that the division operation on real numbers is a compound arithmetic operation composed of multiplication and subtraction. The isomorphism assures that the operations in the domain and range of the isomorphism are equivalent. Consequently, in the SHIAC operation of long division, the multiplication operation is that of spiral multiplication and subtraction is that of the additive inverse of spiral addition. We can demonstrate the application of this algorithm by considering the example of dividing address 1 by address 11 . Armed with the following information, all of the required multiplications and additions for this problem can be determined from Fig. 1(a). Multiplication of addresses by scalars amount to $60^{\circ}$ rotations of the address about the origin

$$
1 1 ^ { - 1 } = 1 1 \longdiv { 1 0 0 0 0 } = 1 . 4 1 4 \ldots .
$$

It is also a straightforward calculation to show that this is the multiplicative inverse as follows:

$$
11 \otimes 11^{-1}=11 \otimes 1.414=1
$$

where $\otimes$ represents the multiplication operator in SHIAC.

Theorem 1 establishes the algebraic validity of performing multiplication and addition of complex numbers in SHIAC. In the following sections, we will demonstrate how the complex computations associated with the Fourier transform can be performed in practice and also demonstrate the computational validity for doing so.

\section{B. Fourier Computations in SHIAC}

An immediate consequence of the isomorphic relation between SHIAC and the complex field is that the arithmetic operations normally performed in the complex field can be performed in SHIAC. Although the motivation for doing so in the infinite SHIAC is of theoretical value only, performing these computations in a finite SHIAC can provide computational advantages by performing computations at a degree of precision concomitant with the user's application. However, before we address this issue in Section V, we must first establish the mathematical machinery required to do so.

Equation (2) reveals that the core computation is that of multiplying components of the input signal with the roots of unity in the complex plane. Consequently, we now describe how to generate the roots of unity in SHIAC. This is accomplished by performing the following two steps. First, determine the roots of unity in the complex plane in the usual manner, and, second, find an address in SHIAC that maps to the point via (4), within a desired tolerance of error. An algorithm to achieve this is named decremental search algorithm and is described formally in Section VI.

The algorithm inputs a complex number and a specified tolerance and outputs an address in SHIAC within that specified tolerance. The algorithm emerges naturally from considerations of the mapping defined in (4). The decremental search algorithm can be employed to determine both the SHIAC roots of unity and to map the input signal into SHIAC. For an input signal composed of $N$ points the unit circle is divide into $N$ equally spaced addresses of magnitude one in SHIAC. If the input signal represents light intensities, the values must be transformed into base seven. If the input signal represents a position in Euclidean space whereby the Fourier descriptors are desired, the algorithm must be used to find its corresponding address in SHIAC.

The operation of multiplication of the input signal with the roots of unity represented in SHIAC is, thus, well defined. Moreover, the mapping of (4) can be employed to control the error 
associated with the multiplication of floating point numbers, which we will consider in the following section.

One final point associated with performing the Fourier computations in SHIAC is the interpretation of the inverse Fourier transform. As the input signal was mapped into SHIAC prior to performing the Fourier computations, the results of the inverse Fourier transform must be converted back to its representation in the complex plane. Once again, (4) and the method to compute $\Omega_{y}$ and $\Omega_{x}$ displayed in Fig. 12 can be employed to convert the signal back to the complex plane from which the spectrum and the phase can be obtained.

\section{Case Study in Error Control}

Computations associated with the execution of any Fourier transform on a digital computer embody an approximation. This approximation results from the error incurred in employing the finite precision of a floating point representation of a real number. In practice, the precision provided by modern computer languages far exceeds that which is required for many practical applications. For example, the error incurred in the representation of bank balances by specifying the balance with the precision provided by two decimal places, is acceptable. Likewise, there are many applications where the Fourier transform can be computed with substantially less precision than that available in a standard representation of a floating point number, without compromising its interpretation. Therefore, addressing the question, "What is the least precision in the representation of a real number so that the resulting Fourier transform remains useful?" can provide computational advantages. In this section, we address this question by investigating the computational requirements that must be satisfied in order to control the error embodied in the recovery of a signal transformed by FFT in SHIAC. To this end we first establish the equations resulting from theoretical considerations of SHIAC. Then, under two different scenarios, we apply the equations to establish the computational requirements imposed by the duck image displayed in Fig. 2(d).

The error embodied in a Fourier component computed in SHIAC $(n, m)$ results from the degree to which the SHIAC discrete roots of unity approximate the continuous roots of unity in the complex plane. This approximation can be made to within any desired specification by setting the value of $m$ in (3) appropriately. The magnitude of $m$ determines the Euclidean distance between adjacent points in SHIAC and, thus, determines the maximum amount of error resulting from a product of a root of unity and the signal. The magnitude of $n$ determines the number of times the error in the product is added, namely, $7^{n}$. However, in this case study, we restrict our considerations to only the error produced by the multiplication of the roots of unity and the signal.

Thus, in order to restrict the error in a Fourier computation to be within a given level of tolerable error, $\varepsilon$, the following inequality must be satisfied:

Max Number (Error Rate) $\leq \varepsilon$
Thus, the smallest value of $m$ that satisfies the following equation will guarantee the actual error is less than $\varepsilon$

$$
\operatorname{Max} \operatorname{Number}(\sqrt{7})^{m}<\varepsilon \text {. }
$$

A solution to the above inequality yields

$$
m>-\frac{\log \left(\frac{\varepsilon}{\operatorname{Max} \text { Number }}\right)}{\log (\sqrt{7})} .
$$

We can now use (5) to calculate the required value of $m$ for the cases of the gray-level and color version of the duck displayed in Fig. 2(d). As we wish to recover integer values of light intensities, an acceptable error rate is 0.5 . As the maximum value of a light intensity is 256 for an 8-bit gray-level image, the solution to (5) for the specified level of error yields $m=6$.

In the case of a color image, the maximum value of a light intensity is an integer composed of nine significant digits. In this case the solution to (5) yields $m=19$.

\section{SHIAC on a Rectangular Lattice}

SHIA was originally developed to perform primitive image transformations on a hexagonal lattice. The initial interest in a hexagonal lattice representation of an image resulted from the suspicion that the ubiquitous occurrence of the hexagon in biological vision systems [5], [6] may have a computational base to it. However, the rectangular lattice (as a means to sample an input signal) is also employed in the vision system of many animals. The occurrence tends to be determined by the amount of light available in the animal's environment. That is, in environments where there is an abundance of light, e.g., in the sky, on the earth's surface and in shallow water, the hexagon is used; this enhances the perception of form and color. In environments where light is at a premium, e.g., in caves and underground and in deep water, the rectangular photoreceptor tends to be employed; this enhances the perception of contrast sensitivity [12].

It turns out that the mathematical structure that underpins SHIA is substantially more general than the particular details that link it to the hexagonal lattice. However, commercially available hexagonal image capture devices for digital technology are rare [13]. In the absence of such image capturing technologies, we now describe an extension to SHIA that adapts its mathematical structure to incorporate a rectangular lattice. Fortuitously, the immediate consequence of this extension pertinent to this paper is that the SHIA-FFT as presented in Section III is easily adapted to perform Fourier transforms on signals that are sampled on a rectangular lattice.

At the heart of SHIA's mathematical structure is an algorithm named spiral counting, as described in [14]. This algorithm specifies the location of SHIA's addresses on the lattice (see Fig. 1); the purpose of the specification was to define spiral addition and spiral multiplication in geometrical terms. On the hexagonal lattice, spiral counting is performed in base seven with sixty-degree rotations. Spiral counting is adapted most naturally to a rectangular lattice by changing the base from seven to five and the $60^{\circ}$ rotations to $90^{\circ}$ rotations. The results of this addressing scheme are displayed in Fig. 8, where addresses 0 to 4 define the initial fundamental cluster of the mosaic. The relative 


\begin{tabular}{|l|l|c|c|c|c|}
\hline & & 20 & & & \\
\hline & & & $\begin{array}{c}\mathbf{3} \\
(-\mathbf{1 , 0})\end{array}$ & & 30 \\
\hline & 13 & $\begin{array}{c}\mathbf{2} \\
(\mathbf{0 , - 1})\end{array}$ & $\begin{array}{c}\mathbf{0} \\
(\mathbf{0 , 0})\end{array}$ & $\begin{array}{c}\mathbf{4} \\
(\mathbf{0 , 1})\end{array}$ & \\
\hline 12 & 10 & 14 & $\mathbf{1}$ & 43 & \\
\hline & 11 & & 42 & 40 & 44 \\
\hline & & & & 41 & \\
\hline
\end{tabular}

Fig. 8. Pixel position of the first fundamental cluster (0-4) with their corresponding Cartesian coordinates and indicates center pixel of adjacent clusters (10-40).

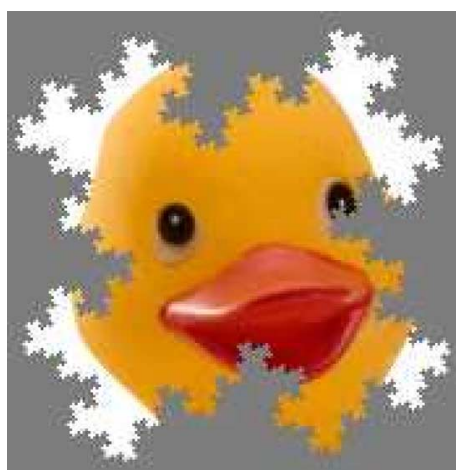

Fig. 9. Image derived from rectangular spiral counting of $5^{6}=15625$ pixels.

positions of addresses $10,20,30$, and 40 specify the recursive step required to enlarge the cluster to five clusters.

An image represented on a mosaic created by the alterations to spiral counting described above is displayed in Fig. 9. The size of the SHIA was chosen so as to emphasize the distinction between the visual world and the visual field. This is an important distinction to make for both computer vision and biological visions systems, as the visual processing unit operates on only a fraction of the total amount of light energy in the environment at any given time.

In particular, the visual world, which is the physical environment where objects move about and where the visual task is to be performed, can be considered as infinite in both the vertical and horizontal directions. It can also be considered infinite in resolution due to the wave nature of light. Because of these properties, the complex plane is an ideal mathematical structure on which to represent the visual world. The visual field is that subset of the visual world bounded by the physical constraints of the camera/eye. The resolution of the visual field can be considered finite as a result of the discrete nature of the photoreceptors of the camera's image sensor or eye's retinal cones. As a result of these properties, the discrete nature of SHIA has advantages over the complex plane as a structure on which to process an image.

It is exactly SHIA's ability to model the visual field that establishes its theoretical importance to computer vision. We will return to this point later in the discussion to strengthen the validity of the claim.

The mosaic is composed of $5^{6}=15625$ pixels. The gray pixels of this figure form the border of the visual field, which gives the appearance of viewing the duck through a fractal-like window.
SHIA's primitive image transformations result from two operations, named spiral addition and spiral multiplication, which are defined in terms of the spiral counting algorithm. With the alterations to Spiral counting mentioned above, the operations of spiral addition and spiral multiplication are defined on the rectangular lattice. As a consequence, all of the primitive image transformations on the hexagonal lattice have their counterpart transformations well defined on the rectangular lattice.

Possibly, the most profound effect of spiral multiplication is the simultaneous scaling up and scaling down of the image. This results in a dual representation of the image after transformation whereby the image is represented at a macro level and simultaneously at a micro level. This effect is important because it is exactly this effect that intrinsically links the SHIA to the Fourier transform. Although this effect of dual representation is present in each of the transformations resulting from spiral multiplication, it is not always perceivable by the human eye. This is due to the relative size of the scaling factor, which can be extreme. However, we can demonstrate this effect with a careful choice of the multiplication address. An address, which possesses a gentle scaling with no rotation, is address 42 . Observe from Fig. 8, that the distance of address 42 from the origin is two pixels. Accordingly, this produces a scaling factor of value 2.0. The macro/micro representation phenomenon is observable in the sequence of images displayed in Fig. 10(a)-(c), which represent the results of repeated applications of transformation M14. The first application produces $5^{1}=5$ subcopies, each of which represents an observable scaling down by a factor of $2^{-1}=0.5$ pixels and a nonobservable (due to magnitude of scaling factor) scaling up by a factor of $2^{6}=64$ pixels. A repetition of M42 on the result of its first application to the original image is displayed in Fig. 10. This image results in a further increase in the number of subimages to $5^{2}=25$, each of which represents an observable scaling down of $2^{-2}=0.25$ of the original image and a nonobservable scaling of $2^{5}=32$. The last image in the sequence represents the 6th application of M14. In Fig. 10, the number of subcopies is $5^{6}=15625$ and a scaling down factor is $2^{-6} \cong 0.015$, which causes the human vision system to perceive the macro level of the image and lose the perception of the micro level.

This switch of perception results from the fact that the scaling is too small for observation. However, the scaling up effect of $2^{1}=2$, is observable, whereby the pixels in the original image of the duck have been spaced by a factor of 2 . This results in a "ghost-like" appearance of the original image of the duck. This dual scaling up and down means that at each representation, resulting from a multiplication operation, the transformed image consists of a macro representation of itself, which is composed of microcopies of itself. Multiplication by address 1 is the trivial case where the macro representation is the original scale and the micro representations are one-pixel representations. All nonunit multiplications produce macro representations that are larger than the original image and micro representations that are composed of more than one pixel. This effect would also be observable in the case where multiplication is with address 10 , which has a scaling factor of $\sqrt{5}$.

The important point about M10 for the rectangular lattice is that it performs exactly the same role as its counterpart transformation on a hexagonal lattice. 


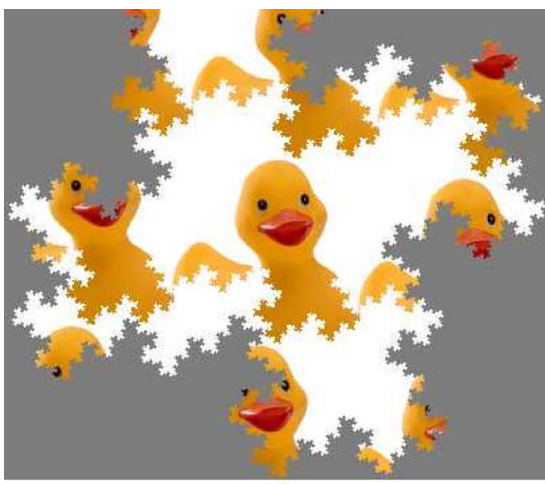

(a)

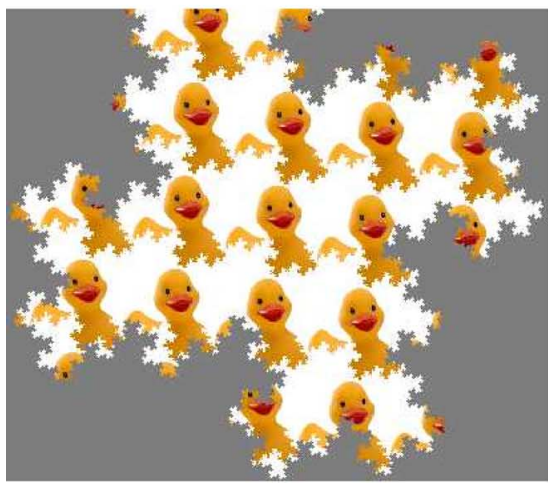

(b)

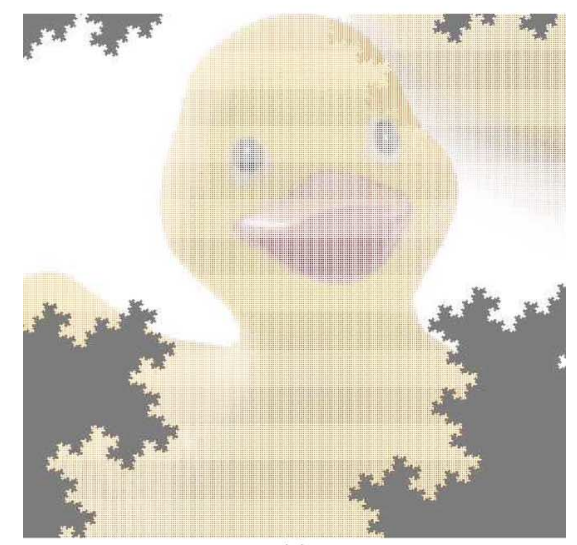

(c)

Fig. 10. (a) Multiplication by address 14 produces five subcopies, each of which represents a scaling by 0.5 of the input image. (b) Multiplication by address 14 of image resolution in Fig. 10(a) produces further replication and scaling. (c) Repeated multiplication by address 14 of image represented in Fig. 10(b), produces a scaling up of image.

As a consequence, the SHIA-FFT as described in Section III, applies equally well to signals sampled by the rectangular lattice.

Moreover, and in accordance with a principal set out in [15], the following observations serve to reveal the deeper relation between SHIA and the DFT. To this end, consider the role that the transform M10 of SHIA plays in connecting the FFT to the DFT. This connection can be expressed at an abstract level as follows:

$$
* \text { Spiral Multiplication }=\text { rotation }+ \text { scaling }
$$

*recursive computation of $\mathrm{FFT}=$ rotation (twiddles) +scaling (FFT on sub- signals).
Another important issue to consider is the mapping, $\Omega$, of rectangular SHIA to Cartesian coordinates.

Equation (4) provides the means by which this mapping can be defined recursively on the row/column contribution to each of the digits in the SHIA representation. The relations between the first five addresses of SHIA and the Cartesian coordinate system, as displayed in Fig. 8, provide the required information to establish the base case of the recursion. Unlike the mapping of the hexagonal SHIA to the Cartesian coordinate system, rectangular SHIA lends itself to a one-to-one mapping in which none of the pixels in the sampled signal need be missed. Fig. 12 displays the details of an algorithm that achieves the desired mapping. This mapping centers the SHIA's origin, address 0 , at the origin of the Cartesian coordinate system, $(0,0)$. To shift the SHIA origin to the center of a $(\mathrm{M} \times \mathrm{N})$ image, $\mathrm{M} / 2$ and $\mathrm{N} / 2$ is added to each of the mapped values under the action of $\Omega$.

As a consequence of the mapping from SHIA to Cartesian coordinates, the size of the SHIA to be used when employing the SHIA-FFT on a standard rectangular $(\mathrm{M} \times \mathrm{N})$ image needs to be considered.

Although the growth in computations in performing a Cooley-Tukey type FFT is $O(n \log n)$ in the size of the image, the size of its domain is a power of its radix. In general, one must be prudent in the choice of the domain size to avoid wastage of computation. Standard image row/column configuration tends to be a power of 2 and so the choice of domain size tends not to be of much concern for a radix-2 FFT. However, the SHIA-FFT is of radix 5 (in the case of the rectangular lattice and 7 in the hexagonal case). This requires the user to make an optimal choice for the domain size. If the entirety of a $(\mathrm{M} \times \mathrm{N})$ image is required, then the minimal choice of the domain must result in wasted computations.

For example, consider the image displayed in Fig. 8, which contains seven columns and six rows. We are interested in determining the smallest SHIA that is sufficiently large enough to contain the forty two pixels. Clearly, a SHIA of size $5^{2}=25<$ 42 is too small. The next smallest SHIA is $5^{3}=125 \gg 42$, which is large enough in this case. However, in general, one must alto that into account the dimensions of the image to be captured. The more the desired image deviates from a square, the more difficult the problem becomes. For example, a 25-pixel SHIA will contain a $4 \times 4$ rectangular image but will not contain an $8 \times 2$ image. In any case, this approach will result in substantial wastage in SHIA pixels. For this problem to be controlled, efficient algorithms need to be designed that can handle sparse matrices.

As an alternative mapping, if only a region of interest is required of the input signal, then the SHIA can be centered appropriately so as to minimize the required number of pixels to be mapped. However, this approach has its own problems. Methods to determine the region of interest are currently ad-hoc.

\section{FFT WITH INTEGER ARITHMETIC}

Having established the mathematical machinery required to perform the Fourier computations in SHIAC, we now return to this issue and describe a method for approximating these computations. The Fourier transform viewed at a higher level of 
abstraction can be described as a weighted average of fundamental patterns derived from the roots of unity in the complex plane. However, the critical properties of these fundamental patterns are not unique to the unit circle. In fact, the patterns can be derived from any circle of a specified radius centered on the origin of the complex plane. We will show that, if one employed a circle whose radius was a function of the number of decimal places required to recover the FFT without error, as described in Section IV.C, then all computations could be performed in SHIA. For example, we have previously established that if the signal to be transformed were an 8-bit gray-level image, it would require six decimal places in SHIAC. This is equivalent to taking a circle whose circumference passes through the address $10^{6}$. The computations of the multiplication of the input signal with these roots would be performed with purely integer addresses in SHIA. The only factor that needs to be adjusted is the division of the sum of the products by the number of points transformed. In particular, the division factor must be multiplied by the radius of the circle to bring the desired weighted average back in line.

Having outlined a method to perform the FFT in SHIA without the need for floating point representation of addresses, we can exploit another computational feature of SHIA that facilitates spiral multiplication at the cost of integer addition. A consequence of the mathematical structure of SHIA is that its set of nonzero addresses can be partitioned into cyclic groups (in the mathematical sense of the word) under the operation of spiral multiplication. If for example, we consider the set of addresses that has as its least significant digit a nonzero digit, the address 12 can be employed as a generator of these addresses. That is, any address, $a$, in this set can be expressed as $a=12^{i}$ for some integer $i$.

The advantage of this representation for addresses of SHIA is that we can employ a computational trick similar to the way multiplication is performed on a slide-rule. That is, each address is stored in an array at a position identified by the index that represents the discreet logarithm of the element. Then, the product of two addresses is achieved in two steps: 1) identify the indices of the position in the array where the elements are stored; 2 ) return the element stored in the position whose index represents the sum of the two indices. With this approach to the multiplication operation of complex numbers, we achieve multiplication at the computational cost of integer addition. We call this representation of SHIA's addresses the multiplicative log-space. The immediate consequence of this Log-Space representation is that multiplication in SHIA corresponds to addition of exponents in the multiplicative log-space.

Exactly the same principle applies to the operation of spiral addition. That is, the set of SHIA addresses from a cyclic group under the operation of spiral addition where address 1 acts as generator of the group. Likewise, each address in SHIA can be represented as an additive power of the address 1 , the consequence of which is that spiral addition in SHIA corresponds to integer addition in the additive log-space representation of SHIA. For full details of the log-space representation of SHIA, the reader is referred to [2].

We can apply these multiplicative and additive log-space representations to obtain computational advantage for the FFT in certain circumstances. All addresses of the SHIA can be held in memory so that their positions in memory reflect their positions in the log-space. Then spiral multiplication and additions are achieved by simply adding the positions of the inputs to obtain the position of the product/sum in memory.

This method of performing the Fourier computations with integer addition alone has its limitations. This method constitutes a tradeoff between processing time and the amount of memory required.

In circumstances requiring a lot of decimal precision, such as in the case of the color image representation as discussed in Section IV-C, the memory requirements would be prohibitive. However, in the case of the 8-bit gray-level image, the memory requirement is quite modest. In general, the ability to exploit the computational potential of this approach is directly related to the complexity of the signal. Consequently, before attempting to employ this method, an analysis of the required precision, such as described in the case study of Section IV-D, is necessary, followed by a decision as to whether the tradeoff to memory utilization is acceptable.

\section{Formal Statement of Algorithms}

This section presents a formal statement of critical algorithms discussed in the paper. In particular, Fig. 11 displays a formal statement of SHIA-FFT and is presented in a MatLab style language as adopted in [15]. Also, the convention of not including the scaling factor for the FFT [15], is also adopted. Fig. 12 displays a formal statement of an algorithm that achieves a mapping from SHIA to the complex plane. Fig. 13 displays the decremental search algorithm.

\section{CONCLUSION}

This paper presented a new methodology for performing a Cooley-Tukey type FFT, named SHIA-FFT. The key to this approach is the employment of a lossless image transformation, named M10, of scaling and rotation from the SHIA. A property of M10 that is crucial to the FFT algorithm presented is its ability to represent an image at the macro level and micro level simultaneously. It is fundamentally this novel property that facilitates the efficient regrouping of the computations required to achieve a FFT as reported in this paper. It was shown that SHIA-FFT achieves considerable computational savings from its ability to perform the arithmetic operations of complex multiplication and complex addition via conventional integer addition by approximating the computation with the Log-space representation of SHIA. Although the computation savings are at the cost of an approximation, it was shown how to determine the required precision to accommodate the user-defined level of acceptable error.

In the process of unveiling this methodology, the paper described two important extensions to SHIA by making minor alterations to the mathematical structure that underpins it. The first extension was shown to be isomorphic to the complex plane. The isomorphism facilitated the description in mathematical terms of the relation between the physical spaces where objects move about (visual world) and that subset of it (visual field) which is sampled by an image capture device at a point in time. 


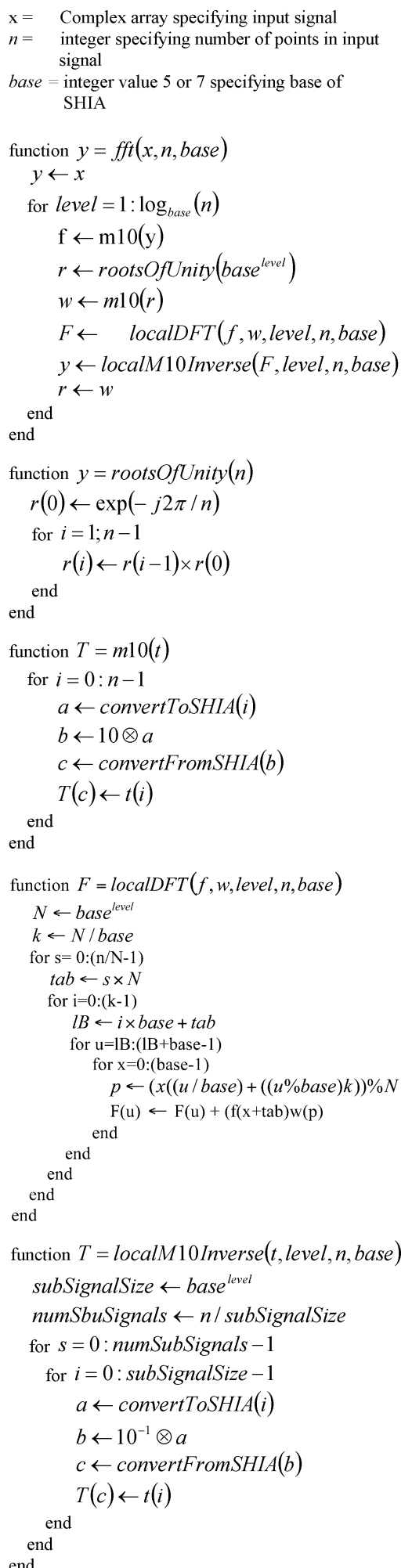

Fig. 11. Formal statement of SHIA-FFT. $\otimes$ as defined in (1).

It was also shown that the isomorphism has computational and theoretical consequences for the field of image analysis. From a computational perspective, it facilitated the measurement and, therefore, control of the implicit error embodied in a representation of an image with a discrete mathematical structure such as SHIA.

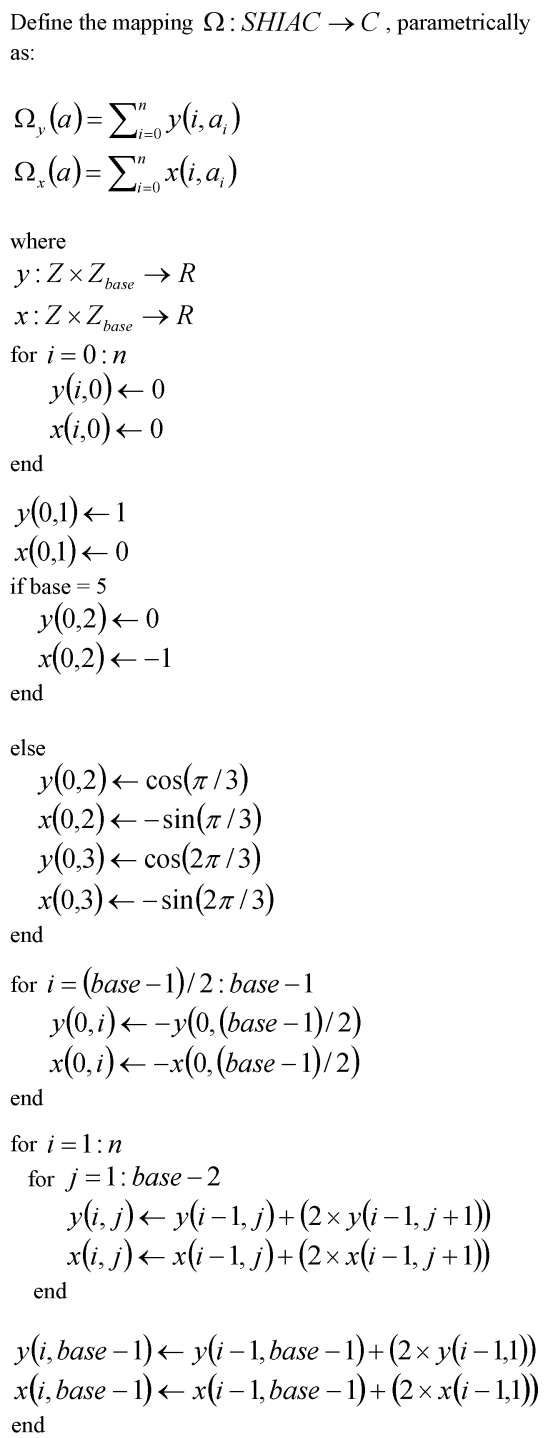

Fig. 12. Mapping from SHIA to the complex plane.

\section{Decremental Search Algorithm}

Input target point and the desired precision.

1. Determine the minimal value of $\mathrm{n}$ such that the magnitude of $10^{n}>$ target point.

2. Let $m=n$.

3. Find the value of $a_{i}$ such that the Euclidean distance from

$$
s=\sum_{i=m}^{n} a_{i} 7^{i}
$$

to the target point is a minimum.

4. If the minimum distance determined in Step 3 is greater than the precision, then decrement $\mathrm{m}$ and repeat Step 3; otherwise return s.

Fig. 13. Decremental search algorithm.

The theoretical importance of the isomorphism is that it relates all of the conventional image analysis algorithms normally performed in the complex plane to SHIA and vice versa. The second extension to SHIA generalized the SHIA-FFT methodology from signals sampled by a hexagonal lattice to signals sampled by a rectangular lattice. 
The immediate pragmatic consequence of this extension is that it enables the application of SHIA-FFT methodology directly to representations obtained by conventional image capture devices without having to first map the rectangular representation to a hexagonal lattice.

In summary, the research presented in this paper advances the practice and theory of image analysis by: 1) describing a new FFT methodology that can be performed on either a hexagonal lattice or rectangular lattice, 2) casting a theoretical framework around this methodology that is in a manner compatible with the natural constraints imposed by image capture devices.

\section{APPENDIX}

\section{A. Section A}

This section provides the proof of SHIA-FFT's correctness.

Notation: The symbol "\%" is employed to denote modular arithmetic

$$
\begin{aligned}
N & =7^{p} \\
n & =N / 7=7^{p-1} .
\end{aligned}
$$

Let e(u) represent the $N$ roots of unity, where

$$
\begin{aligned}
e(u) & =e^{(u \% 7)+(u / 7) n} \\
& =1, e, e^{1}, e^{2}, e^{3}, e^{4}, e^{5}, e^{6}, \ldots, e^{6 n}, e^{6 n+1}, \ldots, e^{6 n+6}
\end{aligned}
$$

for $p>1$ and

$$
=1, e, e^{1}, e^{2}, e^{3}, e^{4}, e^{5}, e^{6}
$$

for $\mathrm{p}=1$

$$
\begin{aligned}
F(u)= & F_{u / 7}^{u \% 7}=F_{0}^{0}, \ldots, F_{0}^{6}, \ldots, F_{n-1}^{0}, \ldots, F_{n-1}^{6} \\
& \text { sequence of } N \text { Fourier components. } \\
f(x)= & f_{x / 7}^{x \% 7}=f_{0}^{0}, \ldots f_{0}^{6}, \ldots, f_{n-1}^{0}, \ldots, f_{n-1}^{6} \\
& \text { sequence of } N \text { points of the input signal. }
\end{aligned}
$$

The proof is by induction on $p$. When $p=1$, the SHIA-FFT is the DFT. Assume the SHIA-FFT computes the Fourier transform for all levels less than $p$

$$
\begin{aligned}
M 10^{-1}\left(f_{x / 7}^{x \% 7}\right)=f_{0}^{0}, \ldots, f_{n-1}^{0}, f_{0}^{1}, \ldots, & \\
& f_{n-1}^{1}, \ldots, f_{0}^{6}, \ldots, f_{n-1}^{6} .
\end{aligned}
$$

We now have seven subimages, each of which is composed of $n$ points. Then by the induction hypothesis, we can apply the FFT algorithm to obtain seven individual transforms of the seven subimages; which yields: Let $\hat{f}=F_{0}^{0}, \ldots, F_{n-1}^{0}, F_{0}^{1}, \ldots, F_{n-1}^{1}, \ldots, F_{0}^{6}, \ldots, F_{n-1}^{6}$. The localized $\hat{f}$ yields

$$
\begin{aligned}
M 10(\hat{f})= & F_{0}^{0}, \ldots, F_{0}^{6}, F_{1}^{0}, \ldots, F_{1}^{6}, \ldots, F_{n-1}^{0}, \ldots, F_{n-1}^{6} \\
M 10(e(u))= & e((u / 7)+(u \%) n) \\
& 1, e^{n}, \ldots e^{6 n}, e^{1} e^{n+1}, \ldots \\
& e^{6 n+1}, \ldots, e^{n-1}, e^{2 n-1}, \ldots, e^{7 n-1} .
\end{aligned}
$$

Perform DFT on each group of seven points. The general term

$$
\begin{aligned}
\text { is } & =0^{-1}\left(\sum_{q=0}^{6} F_{u / 7}^{q} e^{q((u / 7)+((u \% 7) n)) \% N}\right) \\
= & M 10^{-1}\left(\sum_{q=0}^{6}\left[\sum_{r=0}^{n-1} f_{r}^{q} e^{7 r(u / 7) \% N}\right]\right. \\
& \left.\times e^{q((u / 7)+((u \% 7) n)) \% N}\right) \\
= & M 10^{-1}\left(\sum_{q=0}^{6}\left[\sum_{r=0}^{n-1} f_{r}^{q} e^{7 r(u / 7) \% N} e^{(u \% 7) r 7 n \% N}\right]\right. \\
& \left.\times e^{q((u / 7)+((u \% 7) n)) \% N}\right) \\
= & M 10^{-1}\left(\sum_{q=0}^{6}\left[\sum_{r=0}^{n-1} f_{r}^{q} e^{7 r((u / 7)+((u \% 7) n)) \% N}\right]\right. \\
& \left.\times e^{q\left((u / 7)+((u \% 7) n) \%_{N}\right.}\right) \\
= & M 10^{-1}\left(\sum_{q=0}^{6}\left[\sum_{r=0}^{n-1} f_{r}^{q} e^{(7 r+q)((u / 7)+((u \% 7) n)) \% N}\right]\right) \\
= & M 10^{-1}\left(\sum_{s=0}^{N-1} f_{s / 7}^{s} \% e^{s((u / 7)+((u \% 7) n)) \% N}\right) \\
= & M 10^{-1}(F((u / 7)+(u \% 7) n)) \\
= & F(u) .
\end{aligned}
$$

The above proof was written specifically for the hexagonal lattice, base $=7$. However, it applies to the rectangular case, base $=5$, by replacing all occurrences of 7 with 5 and replacing all occurrences of 6 with 4 .

\section{B. Section $B$}

Fundamentally, the arithmetic operations of Spiral Addition and Spiral multiplication are derived from a counting algorithm named Spiral Counting defined in [2]. This counting algorithm evolves naturally in a manner analogous to the way our familiar form of counting underpins the conventional form of addition and multiplication of integers. However, both these counting algorithms are geometrical in nature and so do not lend them to use on a computational device. To achieve a computational definition of the operations we can proceed in the following manner.

The products and sums of basic components for a rectangular lattice as displayed in Tables I and II are derived from interpreting the addresses of Fig. 8 as vectors and the corresponding operations as vector addition and vector multiplication. The corresponding tables for the hexagonal lattice are derived in a similar manner from Fig. 1(a).

Notation: Let base be a variable that takes on the value 5 in the case of a rectangular lattice and 7 in the case of a hexagonal lattice

$$
\begin{aligned}
a_{i}, b_{i}, \alpha & \in[0,1, \ldots, \text { base }-1] \\
a, b & \in \operatorname{SHIAC}(n, m) \\
a & =\sum_{i=-m}^{i=n} a_{i} \operatorname{base}^{i}=a_{n}, \ldots, a_{0}, \ldots a_{-m} \\
b & =\sum_{i=-m}^{i=n} b_{i} \text { base }^{i}=b_{n}, \ldots, b_{0}, \ldots, b_{-m} .
\end{aligned}
$$


TABLE I

DisPlays SPIRAL MultipliCATION ON THE FiRST Fundamental Cluster

\begin{tabular}{|l|l|l|l|l|l|}
\hline$\otimes$ & 0 & 1 & 2 & 3 & 4 \\
\hline 0 & 0 & 0 & 0 & 0 & 0 \\
\hline 1 & 0 & 1 & 2 & 3 & 4 \\
\hline 2 & 0 & 2 & 3 & 4 & 1 \\
\hline 3 & 0 & 3 & 4 & 1 & 2 \\
\hline 4 & 0 & 4 & 1 & 2 & 3 \\
\hline
\end{tabular}

TABLE II

TABLE DisPlays THE SPIRAL ADDITION ON THE FIRST FundAMENTAL CLUSTER

\begin{tabular}{|l|l|l|l|l|l|}
\hline$\oplus$ & 0 & 1 & 2 & 3 & 4 \\
\hline 0 & 0 & 1 & 2 & 3 & 4 \\
\hline 1 & 1 & 42 & 14 & 0 & 43 \\
\hline 2 & 2 & 14 & 13 & 21 & 0 \\
\hline 3 & 3 & 0 & 21 & 24 & 32 \\
\hline 4 & 4 & 43 & 0 & 32 & 31 \\
\hline
\end{tabular}

Spiral addition $a \oplus b$

$$
\begin{aligned}
& \text { carry } \leftarrow 0 \\
& \text { for } \mathrm{i}=-\mathrm{m}: \mathrm{n} \\
& \text { temp } \leftarrow a_{i} \oplus b_{i} \\
& c_{i} \leftarrow \text { temp\%base } \\
& \text { carry } \leftarrow \text { temp/base } \\
& \text { for } \mathrm{j}=\mathrm{i}+1: \mathrm{n} \\
& \text { temp } \leftarrow \mathrm{a}_{\mathrm{j}} \oplus \text { carry } \\
& a_{j} \leftarrow \text { temp\% base } \\
& \text { carry } \leftarrow \text { temp/base. }
\end{aligned}
$$

Scalar spiral multiplication $\otimes$

$$
\alpha \otimes a \leftarrow \alpha \otimes a_{n}, \ldots, \alpha \otimes a_{-m} .
$$

General case of spiral multiplication $a \otimes b$

$$
c \leftarrow 0
$$

for $\mathrm{i}=-\mathrm{m}: \mathrm{n}$

$$
c \leftarrow c \oplus\left(\left(b_{i} \otimes a\right) \text { base }^{i}\right)
$$

$/ /$ now shift the decimal point $m$ places to the left. for $\mathrm{i}=-\mathrm{m}: 2 \mathrm{~nm}$

$c_{i-m} \leftarrow c_{i}$
Example: To illustrate the use of the above algorithms, consider spiral multiplication of $1.2 \times 3.4$

$$
\begin{aligned}
& \otimes \frac{1.2}{4.4} \\
& \oplus \frac{34}{24.11} .
\end{aligned}
$$

Modular Arithmetic in SHIA The special case of SHIAC (n, m) when $\mathrm{m}=0$ permits the definition of a modular form of the arithmetic operations.

Notation: Let $\mathrm{N}=$ base $^{n}$, then we can define in SHIAC (n, 0 ) the following:

$$
\begin{aligned}
& a \oplus b \bmod \mathrm{N} \\
& a \otimes b \bmod \mathrm{N} .
\end{aligned}
$$

For both operations, $\oplus$ and $\otimes$, the arithmetic is performed as specified in the algorithm for each obtaining a value say $\mathrm{p}$. The mod operator is then applied to $\mathrm{p}$ as follows.

If the arithmetic operation is $\oplus$ or the operation is $\otimes$, where the least significant digit of the multiplier is not zero, then the result is simply the $\mathrm{n}$ least significant digits of $\mathrm{p}$, ignoring the others. However, if the least significant digit of the multiplier is zero, then the result is obtained by performing

$$
(\mathrm{p}+(\mathrm{p} / \mathrm{N})) \text { modulo } \mathrm{N} \text {. }
$$

\section{ACKNOWLEDGMENT}

The author would like to thank Prof. B. Thornton, Dr Y. Gao, and V. MacDougall for their helpful comments during the preparation of this manuscript. Software that demonstrates the algebraic features of the Spiral Honeycomb Image Algebra is available at http://www.cit.gu.edu.au/ s999414/research/visiolab.

\section{REFERENCES}

[1] S. Selikoff, Introduction to the Finite Fourier Transform Including a Brief Review of General Fourier Analysis. 2002 [Online]. Available: http://scott.selikoff.net/papers.html

[2] P. Sheridan, T. Hintz, and D. Alexander, "Pseudo invariant transformations on a hexagonal lattice," Image Vis. Comput., vol. 18, no. 11, pp. 907-917, 2000.

[3] Zapata and Ritter, "Fast Fourier transform for hexagonal aggregates," J. Math. Imag. Vis., vol. 12, pp. 183-197, 2000.

[4] L. Middleton and J. Sivaswamy, "The FFT in a hexagonal-image processing framework," in Proc. Image Vision Comput., 2001, pp. 231-236.

[5] W. H. Calvin, "The brain as a darwin machine," Nature, vol. 330, pp. 33-34, Nov. 1987.

[6] D. R. Williams, "Seeing through the photoreceptor mosaic," Trends Neurosci., vol. 9, pp. 193-198, 1986.

[7] X. He, T. Hintz, and Q. Wu, "Neural network based image edge detection within spiral architecture," in Proc. Int. Conf. Imaging Science, Systems, Technology, Las Vegas, NV, 2002.

[8] Q. Wu and J. P. Reilly, "Extension of ESPIRIT method to unknown noise environments," in Proc. Int. Conf. Acoustics, Speech, Signal Processing, 1991, pp. 3365-3369.

[9] Middleton and Sivaswamy, Hexagonal Image Processing. New York: Springer, 2005.

[10] P. Sheridan, "Spiral architecture for machine vision," Ph.D. dissertation, Univ. Technol., Sydney, Australia, 1997, Section 6.2.2, pp. 166-168. 
[11] — , "Spiral architecture for machine vision," Ph.D. dissertation, Univ. Technol., Sydney, Australia, 1997, Appendix A, pp. 179-183.

[12] A. Parker, In the Blink of an Eye. Cambridge, MA: Perseus, 2003.

[13] T. Yamada, K. Ikeda, Y. G. Kim, H. Wakoh, T. Toma, T. Sakamoto, K. Ogawa, E. Okamoto, K. Masukane, K. Oda, and M. Inuiya, "A progressive scan CCD image sensor for DSC applications," IEEE J. Solid-State Circuits, vol. 35, no. 12, pp. 2044-2054, Dec. 2000.

[14] P. Sheridan, "Spiral counting on a rectangular lattice," in Proc. Int. Conf. Parallel and Distributed Processing Techniques and Applications, pp. 383-383.

[15] C. Van Loan, Computational Frameworks for the Fast Fourier Transform. Philadelphia, PA: SIAM, 1992.

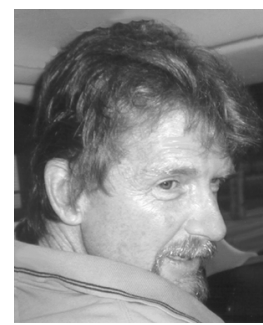

Phil Sheridan (M'98) received the B.A. degree in mathematics from the University of Hawaii, Manoa, the M.Sc. degree in pure mathematics from Monash University, Melbourne, Australia, and the Ph.D. degree in computer science from the University of Technology Sydney, Sydney, Australia, in 1997.

Currently, he is a Senior Lecturer in the School of Information and Communication Technology, Griffith University, Brisbane, Australia. His main research interest is in computer vision and biological vision. 